\title{
Fitting Gravimetric Local and Global Quasi-Geoids to GPS/Levelling Data: The Role of Geoid/Quasi-Geoid Variations
}

\author{
Gomaa M. Dawod $^{(1,2)}$ and Hoda F. Mohamed ${ }^{(1)}$ \\ (1) Survey Research Institute, National Water Research Center, Egypt \\ (2) Currently at Umm Al-Quraa University, Holy Makkah, Saudi Arabia \\ dawod_gomaa@yahoo.com
}

\begin{abstract}
The Global Positioning System (GPS) has been extensively utilized in surveying and mapping applications worldwide and in Egypt, which results in a crucial need for precise geoid models. Traditionally, global and local gravimetric quasi-geoid models are determined and, then, fitted to GPS/leveling data. In the context of precise geoid determination, a quasi-geoid determination has to be converted to a geoid model prior to fitting process. Neglecting such a transforming process leads to the absorption of these effects in the fitting residuals that, hence, may produce misleading results and interpretations. The geoid/quasi-geoid separations in Egypt have been computed and found to range from $-0.074 \mathrm{~m}$ to $0.367 \mathrm{~m}$, with an average of $0.060 \mathrm{~m}$. Additionally, when the quasi-geoid to geoid conversion is performed, it has been found that the undulation of the EIGEN-CG01C global quasi-geoid model when compared to true undulations of HARN stations, have been decreased, in the mean sense, from $0.52 \mathrm{~m}$ to $0.46 \mathrm{~m}$. That means an average improvement of accuracy of about $11 \%$. Based on the obtained results, it can be concluded that the conversion of the quasi-geoid to the geoid is quite significant and it should be a regular step in precise geoid determination in Egypt.
\end{abstract}

Keywords: Quasi-geoid, Geoid Model, Global Geopotential Model, GPS Surveying.

\section{Introduction}

Developing a precise nation-wide geoid model becomes a crucial geodetic task in Egypt in recent decades. It is mainly due to the extensive 
utilization of the Global Positioning System (GPS) technique. The geoid is used to transform the GPS-derived ellipsoidal heights to orthometric heights used in surveying and mapping. Geoid development in Egypt has been addressed by a variety of researchers ${ }^{[1,2,15]}$. The geoid solution of Alnaggar ${ }^{[2]}$ is considered the pioneering nation-wide $\left(22^{\circ} \mathrm{N}<\right.$ latitude $<32^{\circ} \mathrm{N}, 25^{\circ} \mathrm{E}<$ longitude $<37^{\circ} \mathrm{E}$ ) geoid, developed using a least-squares collocation technique and heterogeneous geodetic data including terrestrial gravity, astronomic deflections of the vertical, and Doppler/levelling undulations. A generation of geoid models for Egypt on a five-minute grid has been developed by Saad and Dawod ${ }^{[15]}$ : SRI2001A is a gravimetric geoid computed using local gravity, a local Digital Terrain Model (DTM) and the EGM96 GGM, using a removecompute-restore Fast Fourier Transform (FFT) processing methodology. SRI2001B is a geoid based on surface-fitting of the SRI2001A model to GPS/levelling ${ }^{[15]}$. Abdelmotaal ${ }^{[2]}$ developed and applied a high-degree tailored reference model by merging the available gravity anomalies with the EGM96 Global Geopotential Model (GGM) in order to obtain better residual gravity anomalies. Moreover, there are other studies that have developed geoid models for particular areas of interest in Egypt ${ }^{[19]}$.

Most of gravimetric Egyptian geoids are based on the FFT technique in a remove-restore strategy that utilizes a GGM to represent the long wavelengths of the Earth gravity field. Furthermore, these geoid models are fitted to GPS/levelling data to enhance their integrity and accuracy. A key issue in the utilization of GGMs is that their direct results are the height anomalies (not the geoid undulations) representing a quasi-geoid surface, not the geoid itself. Neglecting the geoid/quasi-geoid separation, among other factors, affect the fitting process between the gravimetric local or global quasi-geoids and the GPS/levelling data ${ }^{[7]}$. This geoid/quasi-geoid transforming step has not been considered in most geodetic literature in Egypt, leading to the fact that the effects of such a correction being absorbed in the fitting residuals that, hence, may produce misleading results and interpretations. The main objectives of the current research study are: (1) the assessment of the geoid/quasigeoid separations, based on real data; and (2) investigating the expected increase of accuracy in fitting quasi-geoids to GPS/levelling data towards the development of a precise geoid model for Egypt. 


\section{Geoid/Quasi-Geoid Separations}

The well-known Stocks' integral for computing geoid undulations from gravity anomalies is ${ }^{[11]}$ :

$$
\mathrm{N}=(\mathrm{R} / 4 \pi \mathrm{G}) \iint_{\sigma} \Delta \mathrm{g} \mathrm{S}(\psi) \mathrm{d} \sigma
$$

Where:

$\mathrm{N}=$ the geoid undulation

$\Delta \mathrm{g} \quad=$ the gravity anomaly

$\mathrm{R}=\mathrm{a}$ mean radius of the earth

$\mathrm{d} \sigma \quad$ denotes the surface element of the unite sphere

$\mathrm{G}=$ a mean gravity value over the earth

$\iint_{\sigma} \quad=$ represents an integral extended over the whole unit sphere

$\mathrm{S}(\psi)=$ the Stoles' function given by ${ }^{[11]}$ :

$$
\begin{aligned}
& \mathrm{S}(\psi)=[1 /(\sin \psi / 2)]-6 \sin (\psi / 2)+1-5 \cos \psi \\
& -3 \cos (\psi / 2) \ln [(\sin (\psi / 2)+\sin 2(\psi / 2))]
\end{aligned}
$$

where

$\psi=\quad=$ the spherical distance between the computational point and the running point.

The remove-restore technique separates three different frequency contributions in the gravity anomalies, so that it may be written that ${ }^{[16]}$ :

$$
\Delta \mathrm{g}=\Delta \mathrm{g}_{\mathrm{FA}}+\Delta \mathrm{g}_{\mathrm{GGM}}+\Delta \mathrm{g}_{\mathrm{H}}
$$

and

$$
\mathrm{N}=\mathrm{N}_{\Delta \mathrm{g}}+\mathrm{N}_{\mathrm{GGM}}+\mathrm{N}_{\mathrm{H}}
$$

where

$\Delta \mathrm{g}_{\mathrm{FA}}=$ the medium wavelength contribution of the local free-air gravity anomalies.

$\Delta \mathrm{g}_{\mathrm{GGM}}=$ the long wavelength contribution as represented by a GGM model.

$\Delta \mathrm{g}_{\mathrm{H}} \quad=$ the short wavelength contribution as represented by the topography or a DTM.

$\mathrm{N}_{\Delta \mathrm{g}} \quad=$ the local gravity medium wavelength component

$\mathrm{N}_{\mathrm{GGM}}=$ the GGM long wavelength contribution. 
$\mathrm{N}_{\mathrm{H}} \quad=$ the topography short wavelength component.

The long wavelength component, as represented by a GGM, can be evaluated in the FFT spherical approximation by ${ }^{[16]}$ :

$$
\mathrm{N}_{\mathrm{GGM}}=\sum_{\mathrm{n}=2}^{\mathrm{n}=\mathrm{ma}} \sum_{\mathrm{m}=0}^{\mathrm{n}} \mathrm{R}\left[\mathrm{C}_{\mathrm{nm}} \cos \mathrm{m} \lambda+\mathrm{S}_{\mathrm{nm}} \sin \mathrm{m} \lambda\right] \mathrm{P}_{\mathrm{nm}}(\sin \phi)
$$

Where:

$\mathrm{C}_{\mathrm{nm}}, \mathrm{S}_{\mathrm{nm}}=$ the fully normalized harmonic coefficients,

$\mathrm{P}_{\mathrm{nm}} \quad=$ the fully normalized associated Legendre polynomial

$\mathrm{n}, \mathrm{m}=$ the degree and order of the GGM model respectively

$\phi, \lambda=$ the geocentric latitude and longitude respectively

GGMs, when used in a spherical harmonic expansion, produce quasi-geoid (sometimes called co-geoid) not geoid solutions since the processing yields height anomalies not geoid undulations ${ }^{[7,10,20]}$. Consequently, the conversion of the obtained quasi-geoid (height anomaly) to the geoid (geoidal undulation) is a regular step in precise geoid determination worldwide ${ }^{[6,8,9,12]}$. Neglecting this procedure, among other factors, affect the fitting process between the gravimetric local or global quasi-geoids and the GPS/levelling data ${ }^{[7]}$. Several researchers have considered this issue and have presented solutions to convert height anomalies to geoid heights. Rapp ${ }^{[13]}$ suggested that potential coefficient models be used first to calculate a height anomaly and then a correction term, represented by a high degree spherical harmonic expansion, be applied to give the geoid undulation. Heiskanen and Moritz ${ }^{[11]}$ provide a formula for computing the geoid/quasi-geoid separation as:

$$
\mathrm{N}=\xi+\left[\left(\mathrm{g}^{\prime}-\gamma^{\prime}\right) / \gamma^{\prime}\right] \mathrm{H}
$$

Where:

$\mathrm{N}=$ the geoid undulations.

$\xi=$ the height anomaly or undulations of quasi-geoid.

$\mathrm{g}^{\prime}=$ the mean gravity along the plumb line between geoid and ground.

$\gamma^{\prime}=$ the mean normal gravity along the normal plumb line between ellipsoid and telleroid.

$\mathrm{H}=$ the orthometric height

For an average density $=2.67 \mathrm{~g} / \mathrm{cm} 3, \mathrm{~g}^{\prime}$ can be computed as ${ }^{[11]}$ :

$$
\mathrm{g}^{\prime}=\mathrm{g}(\text { in gal })+0.0424 \mathrm{H}(\text { in } \mathrm{km})
$$


where: $\mathrm{g}=$ the measured surface gravity.

Additionally, the quantity $\gamma^{\prime}$ may be replaced by a constant average value $^{[11]}$. The maximum geoid/quasi-geoid separation $(\mathrm{N}-\xi)$ can be up to $2 \mathrm{~m}^{[17]}$. In the Mt. Blanc area in the Alps, where the height equals 4877 $\mathrm{m}$, this separation equals $1.8 \mathrm{~m}^{[11]}$. Consequently, applying such a transformation is crucial in the context of developing a precise geoid model for Egypt.

\section{Available Data}

The local geodetic data used in this study was held in two databases (Table 1). Firstly, terrestrial gravity values have been measured at 1138 points (Fig 1). The date of these observations and their accuracy vary greatly between the most recent Egyptian National Gravity Standardization Network of 1997 (ENGSN97) that was established by the Survey Research Institute ${ }^{[3]}$ and older gravity surveys carried out by many private organizations several decades ago. The accuracy of ENGSN97 gravity values is $0.022 \mathrm{mGal}$, while the accuracy estimate for older gravity data is $0.5 \mathrm{mGal}$ on average ${ }^{[4]}$. Even though the accuracy of the older gravity data is not of high-precision, this data set is used in this research study since it covers areas where no other gravity sources are available. As can be seen from Fig. 1, gravimetric data distribution is not homogeneous over Egypt, with significant gaps, particularly in the Eastern and Western deserts ${ }^{[4]}$. However, this data set almost constitute all the published gravity data in Egypt. An evaluation of the existing gravity data, based on comparing the gravity value of each point to values at the nearest four stations, was carried out to identify any large discrepancies. This process identified 58 points (about 5\%) as suspected outliers and these were subsequently removed. The statistics of the remaining 1080 gravity stations are presented in Table 2.

The second compiled geodetic data base consists of $15 \mathrm{GPS} /$ levelling points (Fig. 2). This data set belongs to the High Accuracy Reference Network (HARN), which furnishes the national Egyptian GPS datum established by the Egyptian Survey Authority (ESA) in 1995. The precision of the HARN network is 0.1 part per million (ppm), which can be written in another form as 1: 10,000,000. Although the HARN network consists of 30 stations, only those 15 points have observed real orthometric heights, and consequently true geoid undulations. The 
precision of geoid undulations at these stations may be estimated as $1 \mathrm{~cm}$ or better ${ }^{[3]}$. It is worth mentioning that this data set is quite few in number, but it is of special importance since it is the most accurate and officiallypublished GPS/levelling data in Egypt. Other existing GPS/levelling data do not reach such a precision level, and hence may not be appropriate for the comparison in the current research study. The statistics of the geoid undulations over these stations are presented in Table 3.

Table 1. Available local geodetic data.

\begin{tabular}{|c|c|}
\hline Data type & No. of points \\
\hline Terrestrial Gravity: & 150 \\
1. ENGSN97 Network & 988 \\
2. Older gravity points & 15 \\
\hline GPS/levelling HARN & \\
\hline
\end{tabular}

Table 2. Statistics of the available 1080 gravity stations.

\begin{tabular}{|l|r|r|r|}
\hline \multicolumn{1}{|c|}{ Item } & \multicolumn{1}{c|}{ Min } & \multicolumn{1}{c|}{ Max } & \multicolumn{1}{c|}{ Mean } \\
\hline Observed Gravity (mGal) & 978588.29 & 979509.69 & 979089.18 \\
\hline Orthometric Heights (m) & -253.23 & 1433.52 & 169.34 \\
\hline
\end{tabular}

Table 3. Statistics of the available 15 GPS/Levelling stations.

\begin{tabular}{|l|c|c|c|}
\hline \multicolumn{1}{|c|}{ Item } & Min & Max & Mean \\
\hline Orthometric Heights (m) & 11.76 & 556.42 & 146.49 \\
\hline True Undulations (m) & 10.86 & 21.14 & 15.47 \\
\hline
\end{tabular}

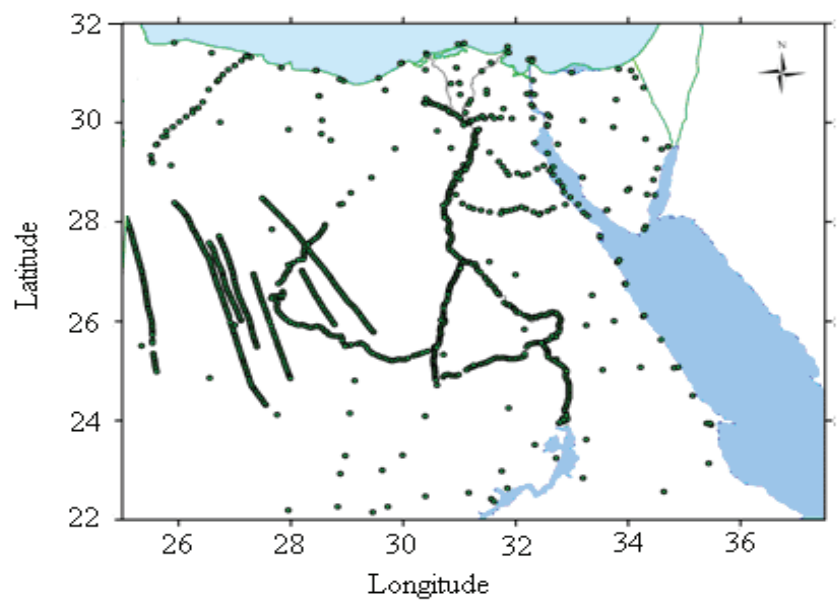

Fig. 1. Available local gravity stations. 


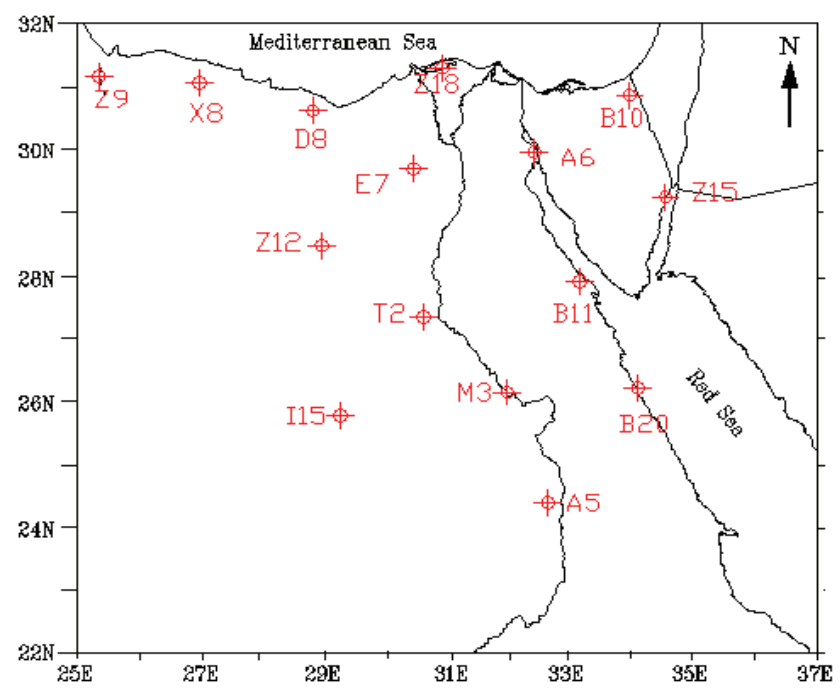

Fig. 2. Available stations with known geoid undulations.

\section{Processing and Results}

The main objectives of the current research study in the assessment of the geoid/quasi-geoid separations, and investigating the expected increase of accuracy in fitting quasi-geoids to GPS/levelling data towards the development of a precise geoid model for Egypt Therefore, the data processing involves two steps. Firstly, the geoid/quasi-geoid separations, through Equations 6 and 7, have been computed at the available known 1080 gravity stations. The attained results are presented in Table 4, and a contour map is depicted in Fig. 3. It can be seen that the geoid/quasigeoid separations range from $-0.074 \mathrm{~m}$ to $0.367 \mathrm{~m}$, with an average of $0.060 \mathrm{~m}$ and a standard deviation equals $0.049 \mathrm{~m}$. That means that such a correction to the gravimetric quasi-geoids can reach up to 37 centimeters at mountainous areas in Egypt (actually this value occurred at Saint Katreen area, south of Sinai peninsula). Moreover, although the topography of Egypt is fairly flat in general, the geoid/quasi-geoid correction has an average value of 6 centimeters. This value is quite significant when a precise geoid model is concerned. 
Table 4. Statistics of geoid/quasi-geoid separations at known 1080 gravity stations (m).

\begin{tabular}{|l|l|l|l|}
\hline Min & Max & Mean & RMS \\
\hline-0.074 & 0.367 & 0.060 & 0.049 \\
\hline
\end{tabular}

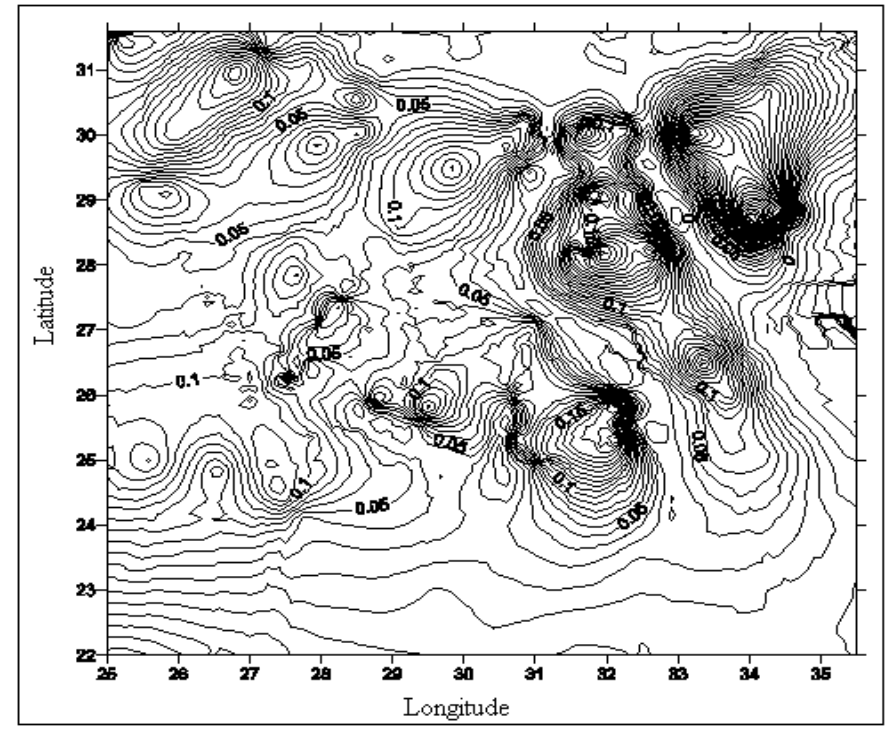

Fig. 3. Geoid/quasi-geoid separation contour map.

The second step in the data processing is the assessment of the expected accuracy of fitting quasi-geoids to GPS/levelling data. It should be emphasized that neglecting the geoid/quasi-geoid separations will be absorbed in the residuals of the fitting process, which causes misleading

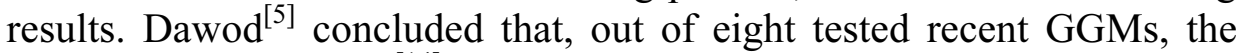
EIGEN-CG01C model ${ }^{[14]}$ is best at representing the long and medium wavelengths of the gravity field in Egypt. Therefore, the height anomaly at the known 15 HARN GPS/levelling stations has been computed using the EIGEN-CG01C GGM, through the SHS360 program (Spherical Harmonic Solution to degree and order 360 computer program ${ }^{[18]}$. The corresponding geoid/quasi-geoid separations at these stations have been interpolated from the contour map. Furthermore, the geoid undulations have been computed by adding the geoid/quasi-geoid separations to the EIGEN-CG01C height anomalies. Two comparisons, of both height anomalies and GGM-based geoid undulations against known geoid undulations $(\mathrm{N}=\mathrm{h}-\mathrm{H})$, have been carried out. The achieved results are tabulated in Table 5. 
Table 5. Statistics of height anomaly and geoid undulations at the known 15 GPS/levelling stations (m).

\begin{tabular}{|l|c|c|c|c|}
\hline \multicolumn{1}{|c|}{ Item } & Min & Max & Mean & RMS \\
\hline EIGEN-CG01C height anomaly & 10.98 & 20.26 & 14.95 & 2.43 \\
\hline $\begin{array}{l}\text { True geoid undulation - EIGEN-CG01C height } \\
\text { anomaly }\end{array}$ & -4.82 & 4.86 & 0.52 & 2.42 \\
\hline Geoid/quasi-geoid separation & 0.002 & 0.16 & 0.06 & 0.05 \\
\hline EIGEN-CG01C geoid undulation & 11.00 & 20.28 & 15.01 & 2.42 \\
\hline $\begin{array}{l}\text { True geoid undulation - EIGEN-CG01C geoid } \\
\text { undulation }\end{array}$ & -4.84 & 4.86 & 0.46 & 2.41 \\
\hline
\end{tabular}

It can be seen that the height anomalies of the EIGEN-CG01C GGM range have a mean value equals $14.95 \mathrm{~m}$ and a standard deviation of 2.43 $\mathrm{m}$. When compared against true geoid undulations, the differences' $\mathrm{s}$ average equals $0.52 \mathrm{~m}$ and a standard deviation equals $2.42 \mathrm{~m}$. The interpolated geoid/quasi-geoid separations at those stations range have a mean value equals $0.06 \mathrm{~m}$ and a standard deviation of $0.05 \mathrm{~m}$. After the quasi-geoid to geoid conversion, the corresponding EIGEN-CG01Cbased geoid undulations have a mean value of $15.01 \mathrm{~m}$ and a standard deviation equals $2.42 \mathrm{~m}$. The computed undulation differences, between the GGM and true values, now have a an average of $0.46 \mathrm{~m}$ and a standard deviation equals $2.41 \mathrm{~m}$. Consequently, the mean difference value of geoid undulation, of the EIGEN-CG01C GGM, has been decreased from $0.52 \mathrm{~m}$ to $0.46 \mathrm{~m}$, which means an average improvement of accuracy of $11 \%$ approximately. Additionally, as presented in Table 4, the geoid/quasi-geoid separations can reach up to 37 centimeters at mountainous areas and their average value, over Egypt, is 6 centimeters which is quite significant when a precise geoid model is concerned. Thus, it should be realized that the utilized known HARN stations exist mostly at flat or moderate-topography areas, and it is expected that the attained findings may be greatly changed for mountainous areas in Egypt. As mentioned previously, the main objective of the current research study is assessing the role of geoid/quasi-geoid separations within the context of developing a precise geoid model. In many geodetic literature, particularly in Egypt, this surface transformation process is not applied in geoid determination. Hence, the attained results highlights, even in a relative sense, the importance of such a processing step that must be taken into account in geoid solutions. 


\section{Summary and Conclusions}

The extensive utilization of GPS in surveying and mapping applications in Egypt has necessitated the development of a precise geoid model. Global and local gravimetric geoid determinations utilize GGMs, in the FFT spherical approximation processing, produce height anomalies representing a quasi-geoid surface, not the geoid itself. The geoid/quasigeoid transforming step has not been considered in most geodetic literature in Egypt, leading to the fact that the effects of such a correction being absorbed in the fitting residuals that, hence, may produces misleading results and interpretations. In order to evaluate the geoid/quasi-geoid separation in Egypt, 1080 gravity stations have been utilized. Although, the gravimetric data distribution is not homogeneous over Egypt, with significant gaps, this data set almost constitute all the published gravity data in Egypt. The orthometric height of these stations, which is the key factor in geoid/quasi-geoid conversion, vary from $-253.23 \mathrm{~m}$ to $1433.52 \mathrm{~m}$, with a mean value of $169.34 \mathrm{~m}$. The attained results show that the values of the quasi-geoid-to-geoid transformation range from $-0.074 \mathrm{~m}$ to $0.367 \mathrm{~m}$, with an average of $0.060 \mathrm{~m}$ and a standard deviation equals $0.049 \mathrm{~m}$. It should be highlighted that this maximum value is quite significant in precise geoid modelling. Hence, it can be concluded that this conversion step is substantial when a precise Egyptian geoid model is concerned.

The second utilized dataset consists of 15 HARN GPS/levelling points, whose true geoid undulations are known. Even though, this data set is quite few in number, but it is of special importance since it is the most accurate and officially-published GPS/levelling data in Egypt. Other existing GPS/levelling data do not reach such a precision level, and hence may not be appropriate for the comparison in the current research study. This data set has been used to investigate the expected accuracy of fitting a global quasigeoid GGM model to GPS/levelling points. The EIGEN-CG01C has been found to be the best GGMs at representing the long and medium wavelengths of the gravity field in Egypt ${ }^{[5]}$. The EIGEN-CG01C height anomalies at those stations have been computed and the corresponding geoid/quasi-geoid separations have been interpolated. Two comparisons, of both GGM-based height anomalies and geoid undulations against known geoid undulations, have been carried out. The results show that when compared the quasi-geoid GGM against true geoid undulations, the differences have an average of $0.52 \mathrm{~m}$. 
After the quasi-geoid to geoid conversion, the corresponding undulation differences have been decreased, in the mean sense, to $0.46 \mathrm{~m}$, which means an average improvement of accuracy of $11 \%$ approximately. Nevertheless, it should be recognized that the exploited HARN stations exist generally at flat or moderate-topography areas, and it is anticipated that the accomplished findings may be greatly changed for mountainous areas. Based on the available utilized data sets and the obtained results, it can be concluded, in a sense, that the conversion of the quasi-geoid to the geoid is quite significant and it should be a regular step in precise geoid determination in Egypt.

\section{References}

[1] Abdelmotaal, H., High-Degree Geopotential Model Tailored to Egypt, Presented at The $1^{\text {st }}$ International Symposium of the International Gravity Field Service (IGFS), Istanbul, Turkey, August 28 - September 1 (2006).

[2] Alnaggar, D., Determination of the Geoid in Egypt Using Heterogeneous Geodetic Data, Ph.D. Dissertation, Cairo University, Egypt (1986).

[3] Dawod, G., A national gravity standardization network for Egypt, Ph.D. Dissertation, Shoubra Faculty of Engineering, Banha Branch, Zagazig University, Cairo, Egypt (1998).

[4] Dawod, G. and Alnaggar, D., Quality control measures for the Egyptian National Gravity Standardization Network (ENGSN97), Proceedings of the Second International Conference on Civil Engineering, Helwan University, Cairo, Egypt, April 1-3, pp: 578-587 (2000).

[5] Dawod, G., Towards the redefinition of the Egyptian geoid: Performance analysis of recent global geoid and digital terrain models, Journal of Spatial Science, 53(1): 31-42, June (2008).

[6] Denker, H., Evaluation and Improvement of the EGG97 Quasigeoid Model for Europe by GPS and Leveling Data, In: M. Vermeer, and J. Adam (Ed.), Proceedings of the Second Continental Workshop on the Geoid in Europe, Budapest, Hungary, March 10-14 (1998).

[7] Featherstone, W., Fitting geoids to GNSS-levelling: You're fooling yourself and fooling others, The Geodetic Week 2006, The International Congress Center Munich (ICM), October 10-12. Munich, Germany (2006).

[8] Featherstone, W., Claessens, S., Kuhn, M. , Kirby, J. and Sproule, D., Progress towards the new Australian geoid-type model, Proceedings of SSC the National Biennial Conference of the Spatial Sciences Institute, Hobart, Australia, May (2007).

[9] Iliffe, J., Ziebart, M., Cross, P. Forsberg, R. Strykowski, G. and Tscherning, C., OSGM02: A new model for converting GPS-derived heights to local height datums in Great Britain and Ireland, Survey Review, 37( 290): 276-293 (2003).

[10] Ivan, M., On the difference between quasi-geoid height and geoid undulation, International Geoid Service Bulletin, 8(December): 53-64 (1998).

[11] Heiskanen, W. A. and Moritz, H., Physical Geodesy, Freeman, San Francisco (1967).

[12] Prijatna, K., A local quasigeoid determination approach for Jawa Island, Indonesia, Presented at the Third FIG Regional Conference, Jakarta, Indonesia, October 3-7 (2004).

[13] Rapp, R., Use of potential coefficient models for geoid undulation determinations using a spherical harmonic representation of the height anomaly/geoid undulation difference, Journal of Geodesy, 71(5): 282-289 (1997).

[14] Reigber, C., Schwintzer, P., Stubenvoll, R., Schmidt, R., Flechtner, F., Meyer, U., König, R., Neumayer, H., Förste, C., Barthelmes, F., Zhu, S., Balmino, G., Biancale, 
R., Lemoine, J. M., Meixner, H. and Raimondo, J., A high resolution global gravity field model combining CHAMP and GRACE satellite mission and surface data: EIGEN-CG01C; Scientific Technical Report STR06/07, GeoForschungsZentrum Potsdam (2006).

[15] Saad, A., and Dawod, G., A precise integrated GPS/gravity geoid model for Egypt, Civil Engineering Research Magazine (CERM), Al-Azhar University, 24(1): 391-405 (2002).

[16] Sideris, M. and Footopoulus, M., Geoid determination by FFT, The International Geoid School, Budapest, Hungary, Jan. 31-Feb. 4 (2005).

[17] Tenzer, R., Vanicek, P., Santos, M., Featherstone, W. and Kuhn, M., The rigorous determination of orthometric heights, Journal of Geodesy, 79(1-3): 82-92 (2005).

[18] Tscherning, C.C., On the chain-rule method for computing potential derivatives, Manuscripta Geodaetica, 1: 125-141 (1976).

[19] Tscherning, C.C. Radwan, A, Tealeb, A., Mahmoud, S., Mohamed, A., Hassan, R., Issawy, E. and Saker, K., Local geoid determination combining gravity disturbances and GPS/levelling: A case study in the Lake Naser area, Aswan, Egypt, Journal of Geodesy, 75: 343-348 (2001).

[20] Tscherning, C.C., Arabelos, D. and Strykowski, G., The 1-cm geoid after GOCE, In: M. Sideris (Ed.): The IAG Symposia, V. 12, pp: 267-270, Springer Verlag (2001). 


\section{تو افق نماذج الجيويد وشبيه الجيويد المحلية و العالمية مع قياسات النظام العالمي لتحديد المو اقع و الميز انيات: دور التغير ات بين الجيويد وشبيه الجيويد}

\section{جمعة محمد داود' 'rو هدي فيصل محمد' \\ 'معهز بحوث المساحة - المركز القومي لبحوث المباه - مصر ' جامعة أم القرى - مكة المكرمة - المدلكة العربية السعودية}

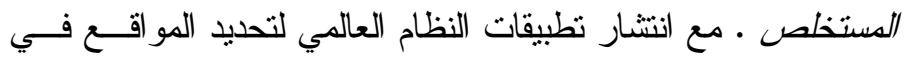

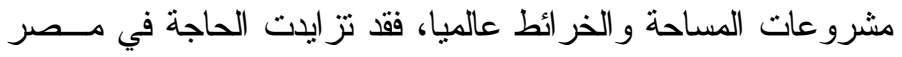

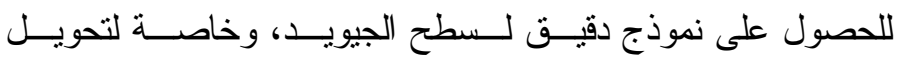

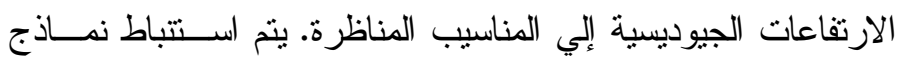

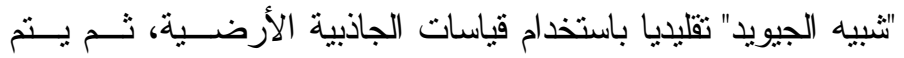

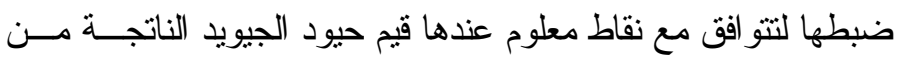

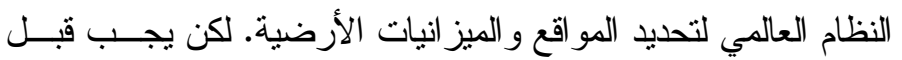

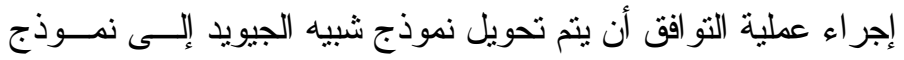

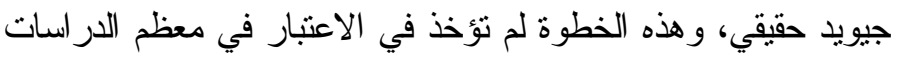

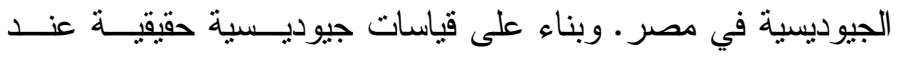

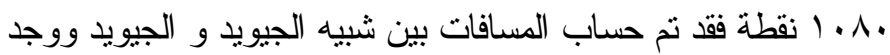

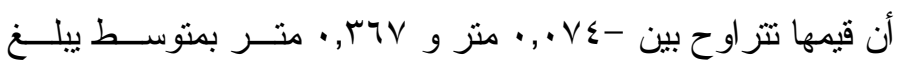

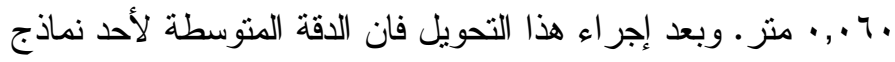

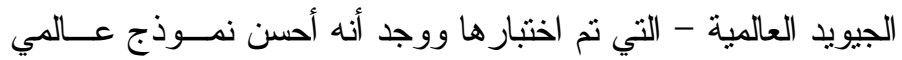

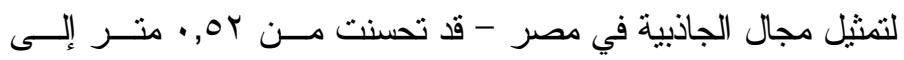

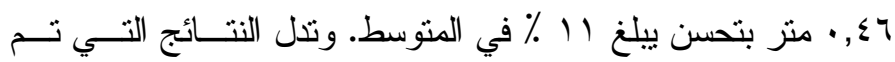

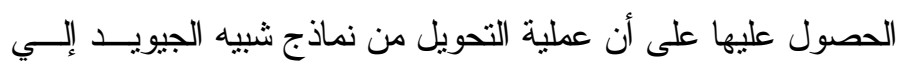

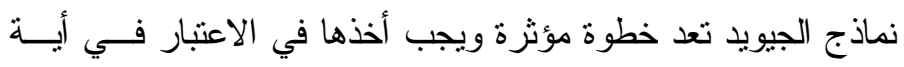

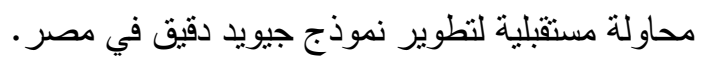

